\title{
3D-conformal radiotherapy for inoperable non-small-cell lung cancer - A single centre experience
}

\author{
Sabine Fromm ${ }^{1}$, Andrea Rottenfusser ${ }^{1}$, Daniel Berger ${ }^{1}$, Robert Pirker ${ }^{2}$, \\ Richard Pötter1, Boris Pokrajac ${ }^{1}$ \\ ${ }^{1}$ Department of Radiotherapy and Radiobiology, Medical University of Vienna, Austria \\ ${ }^{2}$ Department of Internal Medicine, Division of Oncology, Medical University of Vienna, Austria
}

Background. The purpose of this investigation was to evaluate feasibility, safety and efficacy of 3Dconformal radiotherapy (3D-RT) for inoperable non-small-cell lung cancer (NSCLC). Time to progression (TTP), including local recurrence and/or distant metastasis, local control rate (LCR), time to death (TTD) and side effects were evaluated.

Patients and methods. From 1997 to 2002, a total of 84 patients with inoperable NSCLC were treated with 3D-RT according to a prospective protocol at our institution. Depending on performance status, lung function and dose-volume constraints, radiation doses of either 66-70 Gy or 50-60 Gy +/- platin-based chemotherapy were applied.

Results. The treatment was well tolerated and the rate of side effects was low. Only one grade 4 pneumonitis was observed, the rate of grade 3 pneumonitis was $6 \%$ and $13 \%$ for grade 2 . Two patients developed a grade 4 oesophagitis and no grade 3 oesophageal toxicity was observed. The analysis of dose-volume histograms (DVH) found a mean $V_{20}$ (lung volume that receives $20 \mathrm{~Gy}$ ) for the ipsilateral lung (IL) of $42 \%$, a mean $V_{20}$ for the contralateral lung (CL) of $14 \%$ and a mean lung dose IL of $25 \mathrm{~Gy}$. The mean $V_{20}$ IL in patients developing a pneumonitis grade 2-4 was 53.3\%. The mean follow-up was 24 month. There was no difference in TTP (median 15 months) in the different treatment groups. Patients receiving higher radiation doses (66-70 Gy) had a benefit in an overall survival (OS) when the additional chemotherapy was applied (28 month vs. 16 month). Local control rates of mean $22 \%$ after 2 years were low.

Conclusions. The application of radiation doses up to $70 \mathrm{~Gy}$ is feasible and safe, also in combination with chemotherapy. Still, the local control and OS is poor. Thus, further trials to investigate the possibility of dose escalation in the lung without increasing lung toxicity significantly, also in more advanced tumour stages, are mandatory.

Key words: carcinoma, non - small - cell lung - radiotherapy; radiotherapy, conformal; radiotherapy planning; computer - assisted

Received 26 September 2007

Accepted 30 September 2007

Correspondence to: Sabine Fromm, M.D., Department of Radiotherapy and Radiobiology, Medical University of Vienna, Währinger Gürtel 18-20, 1090 Vienna; Austria; Phone: +43 140400 2689; Fax: +43 140400 2690; E-mail: sabine.fromm@akhwien.at

\section{Introduction}

In the European Union (EU) lung cancer is the most common cause of death from cancer. The estimated deaths in 1997 were 180000 , representing one-third of the total 
cancer mortality in the EU. ${ }^{1}$ In Austria, the mortality in men is declining since the 1980 , whereas in women the incidence is rising in the last decades. ${ }^{2}$

For patients with oncological and/or functional inoperable non-small-cell lung cancer (NSCLC), 3D-conformal radiotherapy (3D-RT) has become an established treatment modality. The overall survival and distant failure rate can be improved with chemotherapy (ChT), applied sequential or concurrent to conformal radiotherapy. ${ }^{3-5}$ Therefore, combined chemo/radiotherapy is today the standard treatment for patients with advanced inoperable NSCLC in Stage IIIA/IIIB. ${ }^{6}$

Still, the most common pattern of failure is local recurrence. For patients surviving 6-12 months, local tumour control results in the increased overall survival and is directly related to the applied radiation dose. ${ }^{7-9}$ With a radiation dose ranging from 64 to $80 \mathrm{~Gy}$, local control rates of $70-92 \%$ can be achieved for stage T1-T3 tumours over a period of 12 months which decreases dose-dependently to $73-43 \%$ in the following years. Thus, attempts to decrease the rate of intrathoracic recurrence have been concentrated on dose escalation and different fractionation schedules throughout the last years. ${ }^{3,10-13}$

The purpose of this investigation was to evaluate feasibility, safety and efficacy of 3D- conformal radiotherapy with doses up to $70 \mathrm{~Gy}+$ /- platinum-based chemotherapy in patients with inoperable NSCLC treated within a prospective protocol in our institution.

\section{Patients and methods}

This investigation includes 84 patients with oncological and/or functional inoperable, histologically proven NSCLC consecutively treated from 1997 to 2002 in the poten- tial curative intent at the Radiotherapy Department of the Medical University of Vienna. According to a prospective protocol, 3D-RT with radiation doses up to 66-70 Gy was applied, depending on dosevolume-constraints for lung toxicity, performance status and lung function. All patients had a complete staging before the treatment including CT scan of the chest and upper abdomen, bronchoscopy, CTguided biopsy or mediastinoscopy, pulmonary function test, bone scan and complete blood cell counts.

\section{Radiotherapy}

All 84 patients received 3D-conformal radiotherapy. The computed tomography (CT) for the planning was performed in inspiration while the patient was lying in a supine position with arms elevated above the head. During the treatment the patients were asked to hold the breath as maximal as possible. The planning CT scan of the entire thorax was done with a slice thickness of $8 \mathrm{~mm}$. For optimal positioning, the breast-board was used. The CT images were transferred to the 3D planning system $\left(\right.$ HELAX $^{\circledR}$, MMS 6.1B). Organs at risk (lung, spinal cord, heart), gross tumour volume (GTV), clinical target volume (CTV) and planning target volume (PTV) were delineated. The GTV contoured the primary tumour plus involved pathological lymph nodes $(\geq 10 \mathrm{~mm})$. Patients with supraclavicular lymph node metastases were not included.

The CTV was defined by adding $10 \mathrm{~mm}$ around the GTV including also regional lymph nodes (mediastinum and ipsilateral hilus). For the PTV an additional margin of $5 \mathrm{~mm}$ was placed around the CTV. The dose was prescribed to the ICRU point as described in the ICRU 50 recommendations. ${ }^{14}$ Dose volume histograms (DVH) have been calculated for both lungs and spinal cord. 
For the definition of the dose-volume constraints we followed the recommendations of Graham et al. ${ }^{15}$ The maximum 20 Gy volume $\left(\mathrm{V}_{20}\right)$ was tolerated to be $50 \%$ for ipsilateral (IL), 30\% for contralateral (CL) and $40 \%$ for both lungs. The mean lung dose for the ipsilateral lung had not to be more than 25 Gy. The dose to the spinal cord was maximum 50 Gy.

The irradiation was delivered by multiple field arrangements using photons with an energy of 10-25 MV. A dose of 40 Gy was applied by AP/PA fields, followed by a field rearrangement (3-4 fields) to lower the dose to the spinal cord. For boost irradiation to the primary tumour, the shrinking field technique was used. The treatment was performed in conventional fractionation, 5 days a week, with a dose of 2 Gy per fraction.

\section{Chemotherapy}

A platinum-based chemotherapy in doublets was applied mainly sequential. Cisplatin was combined with taxotere, navelbine, etiposide, gemcitabine or ifosfamide. The majority of patients (20) received 3 cycles before radiotherapy. A maximum of 6 cycles, 3 before and 3 after irradiation was given in 7 pts. Only 4 patients had concomitant chemo/radiotherapy (Table 1).

\section{Side effects}

The diagnosis of pneumonitis was based on clinical symptoms of shortness of breath, cough, fever and in correlation with the radiographic findings on chest X-ray. Radiation pneumonitis and oeosphagitis were evaluated according to the RTOG/ EORTC radiation morbidity scoring criteria: ${ }^{16}$ grade 2 pneumonitis is defined as cough, requiring medication or mild dyspnea during exercise, grade 3 as clinical or on X-ray visible acute pneumonitis requiring steroids and maybe intermittent oxygen and grade 4 is severe respiratory insufficiency and continuous use of oxygen. Grade 2 oesophagitis is defined as mild dysphagia requiring medication, grade 3 as severe dysphagia with weight loss and dehydration, grade 4 as complete obstruction, ulceration, perforation or fistula.

\section{Patients}

Depending on DVH constraints for $\mathrm{V}_{20}$ and mean lung dose, forced expiratory volume in 1 second $\left(\mathrm{FEV}_{1}\right)$ and performance status, radiation doses of either 66-70 Gy or 5060 Gy were chosen. The administration of chemotherapy depended on performance status and co-morbidity. Thus, for the retrospective analysis four different treatment groups were defined:

Patients with a Karnofsky-index (KI) of $70-100 \%, \mathrm{FEV}_{1}$ of $\geq 1,31$, treated with 3D-RT in combination with chemotherapy to a total dose of 66-70 Gy were defined as group $\mathrm{A}$ and patients with the same KI and $\mathrm{FEV}_{1}$ but severe co-morbidity, treated with 66-70 Gy without chemotherapy as group C. A poorer performance status and/or a $\mathrm{FEV}_{1} \leq$ 1,3 1 and high values at the DVH analysis resulted in 3D-RT of 50-60 Gy with chemotherapy (group B) and in case of additional severe co-morbidity, 3D-RT (50-60 Gy) was performed without chemotherapy (group D). See details in Table 1.

\section{Follow-up}

After the treatment the patients were followed in a 3 months (mo) interval for the first 2 years (y). A thoracic CT-scan and a pulmonary function test were demanded in regularly intervals. If clinically indicated, a blood test and a CT-scan of the brain were performed. The recurrent disease was defined as appearance of new lesions on CTscan 6 months after radiotherapy. 
Table 1. Patients characteristics

\begin{tabular}{|c|c|c|c|c|}
\hline Group & $\begin{array}{c}\mathbf{A} \\
(66-70 \mathrm{~Gy}+\mathrm{ChT})\end{array}$ & $\begin{array}{c}\text { В } \\
(50-60 \mathrm{~Gy}+\mathrm{ChT})\end{array}$ & $\begin{array}{c}\text { C } \\
(66-70 \text { Gy) }\end{array}$ & $\begin{array}{c}\text { D } \\
(50-60 \mathrm{~Gy})\end{array}$ \\
\hline Age (mean) & $48 \mathrm{y}$ & $61 \mathrm{y}$ & $70 \mathrm{y}$ & $70 \mathrm{y}$ \\
\hline \multicolumn{5}{|l|}{ Sex (n) } \\
\hline Female & 13 & 6 & 5 & 5 \\
\hline Male & 16 & 10 & 21 & 8 \\
\hline \multicolumn{5}{|l|}{ Histology (n) } \\
\hline Squamous cell carcinoma & 12 & 7 & 18 & 8 \\
\hline Adenocarcinoma & 16 & 6 & 6 & 4 \\
\hline Large cell carcinoma & 1 & 3 & 2 & 1 \\
\hline \multicolumn{5}{|l|}{ Stage (n) } \\
\hline IA & & & 1 & \\
\hline IB & & & 5 & 2 \\
\hline IIB & 1 & 1 & 6 & 2 \\
\hline IIA & 5 & 2 & 8 & 5 \\
\hline IIIB & 23 & 13 & 6 & 4 \\
\hline \multicolumn{5}{|l|}{ TNM (n) } \\
\hline T1N0 & & & 1 & \\
\hline T1N2 & 2 & & & \\
\hline T1N3 & 1 & 1 & & \\
\hline T2N0 & & & 5 & 2 \\
\hline $\mathrm{T} 2 \mathrm{~N} 1$ & & & 1 & \\
\hline $\mathrm{T} 2 \mathrm{~N} 2$ & 3 & & 7 & 3 \\
\hline $\mathrm{T} 2 \mathrm{~N} 3$ & 2 & & & 1 \\
\hline T3N0 & 1 & 1 & 4 & 2 \\
\hline T3N2 & 1 & 3 & 2 & 2 \\
\hline T3N3 & 3 & 3 & & \\
\hline T4N0 & 8 & 2 & 4 & 3 \\
\hline T4N1 & & & 1 & \\
\hline T4N2 & 7 & 5 & & \\
\hline T4N3 & & 1 & 1 & \\
\hline TXN3 & 1 & & & \\
\hline
\end{tabular}

\section{RT dose (n; Gy)}

\section{0}

60

66

70
7

9

15

14
7

6

12

15

\section{ChT cycles (n)}

$\begin{array}{lcc}6 & 7 & 2 \\ 5 & 1 & 0 \\ 4 & 3 & 2 \\ 3 & 13 & 8 \\ 2 & 1 & 3 \\ 1 & 0 & 1 \\ ? & 4 & 0\end{array}$

$\mathrm{RT}=$ radiotherapy; $\mathrm{ChT}=$ chemotherapy; $\mathrm{n}=$ number of patients

Radiol Oncol 2007; 41(3): 133-43. 
Table 2. Dose volume histograms (DVH) analysis for all treatment groups (A-D), mean values and range

\begin{tabular}{lcccc}
\hline Group & A & B & C & D \\
& $(66-70 \mathrm{~Gy}+\mathrm{ChT})$ & $(50-60 \mathrm{~Gy}+\mathrm{ChT})$ & $(66-70 \mathrm{~Gy})$ & $(50-60 \mathrm{~Gy})$ \\
\hline V20IL (\%) & 39 & 47 & 39 & 47 \\
& $(23-56)$ & $(25-80)$ & $(10-75)$ & $(37-60)$ \\
V20CL (\%) & 16 & 15 & 14 & 10 \\
& $(2-40)$ & $(0-35)$ & $(1-33)$ & $(1-30)$ \\
Mean lung dose IL (Gy) & 23 & 25 & 27 & 24 \\
& $(16-32)$ & $(9-41)$ & $(10-36)$ & $(15-33)$ \\
Mean lung dose CL & 11 & 11 & 10 & 7 \\
(Gy) & $(4-25)$ & $(4-24)$ & $(3-18)$ & $(3-12)$ \\
\hline
\end{tabular}

$\mathrm{IL}=$ ipsilateral lung, $\mathrm{CL}=$ contralateral lung

\section{Statistical analysis}

Time to progression (TTP) was defined as an interval from the date of diagnosis to the development of local recurrence and/or distant metastasis or date of the last follow-up visit. Local control rates (LCR) of $1 \mathrm{y}$ and $2 \mathrm{y}$ were evaluated. Time to death (TTD), TTP and time to pneumonitis (grade 2-4) were estimated using the Kaplan-Meier product-limit method. To test the difference between survival curves, the log-rank test was used. ${ }^{17}$

\section{Results}

Data have been analysed until November 2004. The evaluation included TTP, TTD, LCR, distant failure and side effects such as pneumonitis and oesophagitis. Additionally a DVH analysis was done. The mean follow up time was 24 mo (range 1-84), 2 patients were lost to the follow-up.

Although DVH were calculated for all patients at the time of treatment, in 11 patients the calculated DVH could not be retrieved retrospectively from the planning system due to a technical defect on the hard disc.
The DVH analysis showed a mean $\mathrm{V}_{20} \mathrm{IL}$ of $42 \%$ with a range of 10 to $80 \%$. The $\mathrm{V}_{20} \mathrm{CL}$ was $14 \%$ (range $0-40 \%$ ). The mean lung dose IL was 25 Gy (range 9-36) and CL 10 Gy (range 3-25). Although the mean values correspond to the dose volume constraints we used, in some patients it was due to the tumour volume not possible to refer to those parameters, what explains the large range of the different values. Thus, patients with higher values of V20 IL and mean lung dose IL and low $\mathrm{FEV}_{1}$ received only 50-60 Gy as already mentioned above (Table 2).

Of all 84 patients, 1 patient (1\%) developed a grade 4 pneumonitis, 5 patients $(6 \%)$ a grade 3 pneumonitis and 11 patients (13\%) a grade 2 pneumonitis (Table 3 ). The median time of the development of pneumonitis in patients who experienced a pneumonitis grade 2-4 was $2 \mathrm{mo}$, ranging from 1 to 11 mo.

The statistic analysis for all patients showed that in a mean observation time of 24 mo (1-84) the median time of pneumonitis was not reached. The proportion of patients developing a pneumonitis grade 2-4 was after 3 mo 15\%, after 6 mo $18 \%$ and after 11 mo 22\%. Patients with a pneumonitis grade 2-4 had a mean $V_{20}$ IL of $53.3 \%$ (33$85)$ and a mean lung dose IL of 30 Gy (17.2- 
Table 3. Incidence of pneumontis and oesophagitis in the different treatment groups

\begin{tabular}{lcccc} 
Group & A & B & C & D \\
Pneumonitis & $(66-70 \mathrm{~Gy}+\mathrm{ChT})$ & $(50-60 \mathrm{~Gy}+\mathrm{ChT})$ & $(66-70 \mathrm{~Gy})$ & $(50-60 \mathrm{~Gy})$ \\
Grade 2 & & & & $3(23 \%)$ \\
Grade 3 & $4(13 \%)$ & $2(11 \%)$ & $3(11 \%)$ & 0 \\
Grade 4 & $2(6 \%)$ & 0 & $2(7 \%)$ & $1(8 \%)$ \\
Oesophagitis & 0 & 0 & 0 & \\
Grade 2 & & & & $1(8 \%)$ \\
Grade 3 & $5(17 \%)$ & $3(17 \%)$ & 0 & 0 \\
Grade 4 & 0 & 0 & 0 & 0 \\
\hline
\end{tabular}

37.5). The additionally calculated sigmoid dose-response curve (Figure 1) showed a risk of $60 \%$ to develop a pulmonary toxicity (> grade 1 ) when the mean $\mathrm{V}_{20}$ IL was more than $50 \%$.

Looking at the detailed results of the different treatment groups, we found in group A a median TTP of 13 mo, with a range of 485 mo and a median TTD was 28 mo, ranging from 7-85 mo. The median disease specific survival (DSS) was also 28 mo. The survival after 3 and $5 y$ was $38 \%$ and 10\% respective-

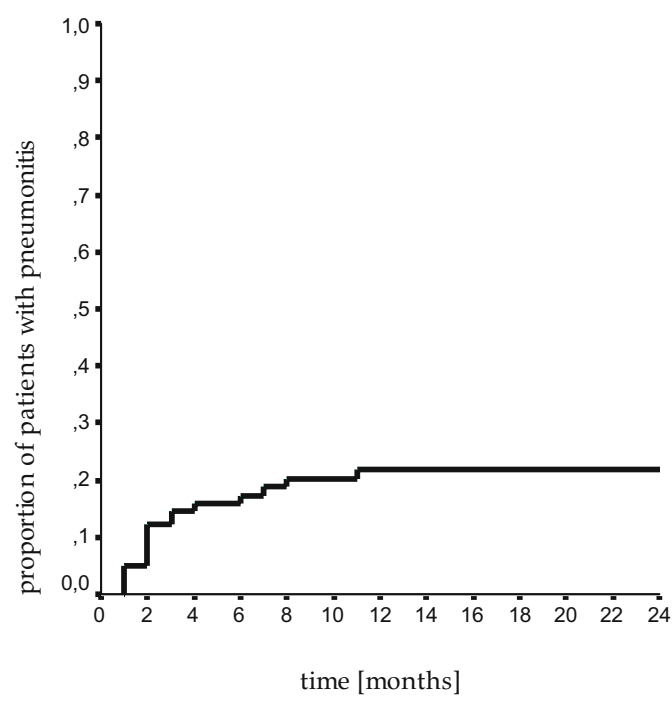

Figure 1. Sigmoid dose-response curve showing the correlation between V20 (\%) IL and probability for pulmonary toxicity more than grade 1 . ly. The LCR was $48 \%$ after 1 y and $24 \%$ after $2 y$. Eight patients (27\%) had progressive disease after radiotherapy, 12 (41\%) developed distant metastases and 2 died of other, not cancer related causes. One patient with a stage IIIB tumour, located centrally, close to the oesophagus and the trachea developed a fistula (grade 4 complication) which was treated by a stent implantation. This patient died shortly after radiotherapy due to the local tumour progression.

In group B, the median TTP was 15 mo ranging from 3-58 mo, the median TTD was 16 mo (3-27 mo), the LCR 38\% after 1y and $25 \%$ after $2 y$. The three and $5 y$ survival was $19 \%$ and $6 \%$ and the median DSS was 16 mo. The progressive disease after irradiation was seen in 5 patients (31\%). A total of 5 patients (31\%) developed distant metastases and 1 died of other causes. One 73y old patient with a T4N2 (stage IIIB) tumour developed an ulceration of the oesophageal mucosa (grade 4 complication) after irradiation, requiring hospitalisation, intravenous fluids and hyperalimentation.

In group $C$ the median TTP was 13 mo (range 5-65 mo), the median TTD $16 \mathrm{mo}$ (range 5-78 mo), the median DSS was 15 mo, the LCR was $42 \%$ after $1 y$ and $23 \%$ after $2 \mathrm{y}$. Nine patients (35\%) had a tumour progression after radiotherapy, 6 (23\%) had distant failure and 8 (31\%) had not cancer 
Table 4. Median values for time to progression (TTP), time to death (TTD), disease specific survival (DSS) and local control rate (LCR)

\begin{tabular}{lcccc}
\hline Group & A & B & C & D \\
& $(66-70 \mathrm{~Gy}+\mathrm{ChT})$ & $(50-60 \mathrm{~Gy}+\mathrm{ChT})$ & $(66-70 \mathrm{~Gy})$ & $(50-60$ Gy $)$ \\
\hline TTP (month) & 13 & 15 & 13 & 13 \\
TTD (month) & 28 & 16 & 16 & 22 \\
DSS (month) & 28 & 16 & 15 & 16 \\
LCR (\%) & & & & 38 \\
1-year & 48 & 53 & 42 & 15 \\
2-year & 24 & 25 & 23 & \\
\hline
\end{tabular}

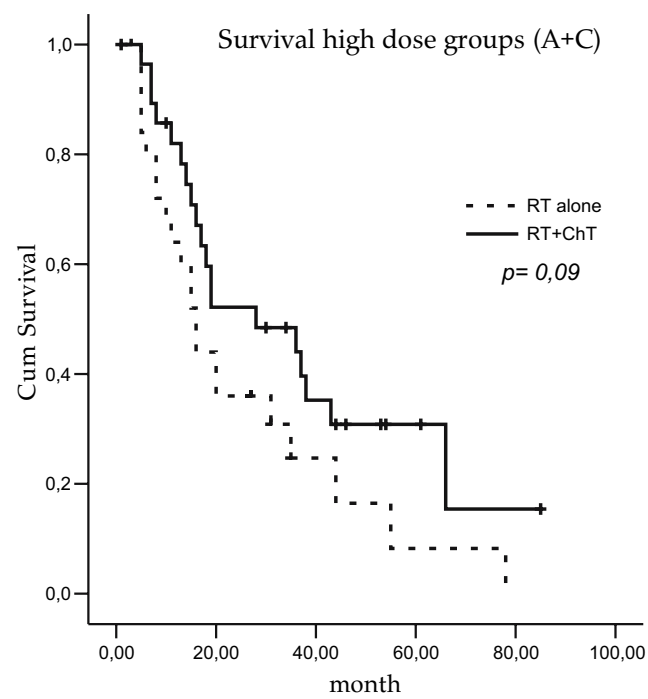

Figure 2. Kaplan-Meier overall survival curve for group A+C (66-70 Gy +/- ChT).

related death. The three and 5y survival was $11 \%$ and $8 \%$. No grade 3 or 4 oesophageal toxicity was found.

Group D had a median TTP of 19 mo (range 3-22 mo), a median TTD of 22 mo (range 3-42 mo) and a median DSS was 16 mo. The LCR was 53\% after $1 \mathrm{y}$ and $15 \%$ after $2 y$. Four patients (31\%) had progressive disease after radiotherapy, no distant metastases were observed. Three patients died of other causes. The survival after 3 and $5 y$ was $23 \%$ and $8 \%$. No grade 3 or 4 oesophagitis was seen. One patient with a

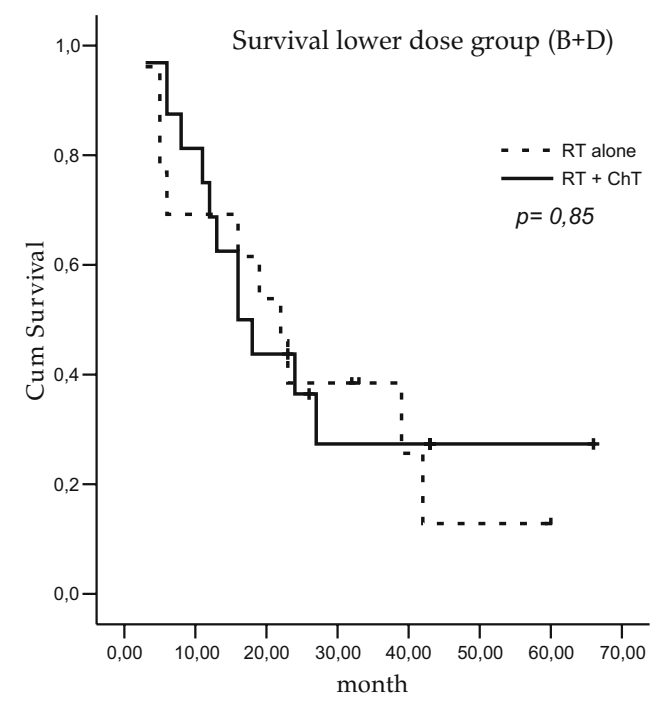

Figure 3. Kaplan-Meier overall survival curve for group B+D (50-60 Gy +/- ChT).

T2N2 (stage IIIA) tumour developed a grade 4 pneumonitis, requiring continuous oxygen. The ipsilateral $\mathrm{V}_{20}$ for this patient was $60 \%$ and the ipsilateral mean lung dose was 32 Gy. Recurrent disease was found after 10 months and death occurred 42 mo after diagnosis, without distant metastases.

\section{Long-time survivors}

For all treatment groups together a total of 14 patients survived more than $2 \mathrm{y}$ with a median survival of $40 \mathrm{mo}$, ranging from 26 
to 61 mo. Concerning long-time survival there was no significant difference between the different treatment groups (Figures 2, $3)$; however, patients receiving higher radiation doses (66-70 Gy) had a benefit in an overall survival (OS) when the additional chemotherapy was applied (28 month $v s$. 16 month).

\section{Discussion}

In the last decades, different treatment strategies were developed for locally advanced non-small cell lung cancer. For inoperable stage III NSCLC, 3D-conformal radiotherapy in combination with chemotherapy has lead to a better local control and longer survival. ${ }^{7}$ Nevertheless, the local control remains poor, although the first results in dose escalation trials are promising. $3,13,18$

All patients included in this retrospective analysis received a $3 \mathrm{D}$-conformal radiotherapy within a prospective protocol with the aim to apply a maximum dose of $70 \mathrm{~Gy}$. Depending on performance status, lung function and dose volume constraints such as calculated mean lung dose and 20 Gy lung volume $\left(\mathrm{V}_{20}\right)$ different radiation doses \pm chemotherapy have been applied on an individual basis resulting in four treatment groups. Our analysis showed that the application of radiation doses up to $70 \mathrm{~Gy}$ also in combination with sequential chemotherapy is feasible and safe. The treatment could be finished in all patients and no treatment related death occurred. Four grade 3 pneumonitis (9\%) were found in the "higher dose" (66-70 Gy) groups $(A+C)$ and no grade 4 pneumonitis.

The DVH analysis emphasised that the $\mathrm{V}_{20}$ IL in patients with grade 2-4 pneumonitis was mean $53.3 \%$, with a mean lung dose IL of $30 \mathrm{~Gy}$. Furthermore, the sigmoid doseresponse curve shows that a $V_{20}$ IL of more than $50 \%$ leads to a risk of $60 \%$ for pulmonary toxicity. This underlines the correlation of $V_{20}$ IL with the incidence of radiation pneumonitis as reported by Graham et al. ${ }^{15}$ The authors showed that a mean $\mathrm{V}_{20}$ of the total lung (including both lungs minus PTV) of more than $32 \%$ was significantly correlating with high grade pneumonitis ( $\geq$ grade 3 ). The final toxicity results of a dose-escalation study of Kong et al. showed that the grade 2 and 3 lung toxicity was not directly associated with the prescribed tumour dose but correlated with dosimetric parameters. The risk of pneumonitis was significantly associated with a mean lung dose $\geq 14$ Gy $(p=0.002)$ and a $V_{20} \geq 27 \%(p$ $=0,0008){ }^{19}$

For all 84 patients, no grade 3 oesophagitis was observed. Two grade 4 oesophageal toxicities were seen in patients with central, bulky tumours close to oesophagus and trachea.

Our results also showed that in the group with higher radiation doses (66-70 Gy) the application of chemotherapy prolonged the survival compared to exclusive radiotherapy of 66-70 Gy (28 vs. 16 mo). Considering the DSS we found the same results (28 vs. 15 $\mathrm{mo})$. Comparable results have been reported by Rengan et al. ${ }^{12}$ In this study a group of 35 patients with stage IIIB NSCLC received 64-84 Gy plus chemotherapy with a median overall survival (OS) of 20 mo for and local failure rate of $64 \%$. Sim et al. ${ }^{5}$ reported for 152 patients with stage III NSCLC a median OS of 18 mo for the combined treatment compared to 11.7 mo for the radiotherapy alone group $(p \leq 0,001)$.

In the lower dose $(50+60 \mathrm{~Gy})$ groups $(\mathrm{B}+\mathrm{D})$ there has been no benefit in OS for patients receiving $\mathrm{ChT}$ as it was seen in the higher dose groups $(\mathrm{A}+\mathrm{C})$. The median survival time for the lower dose groups +/- ChT (16 vs. $22 \mathrm{mo}$ ) is also comparable with results in the literature. Different studies report a median OS of 13.8-16 mo 
for chemoradiotherapy and 9.7-16 mo for exclusive radiotherapy in stage III NSCLC with radiation doses between 50-63 Gy. ${ }^{12,20-22}$ The slightly better results for the group with exclusive radiotherapy (22 mo) for OS could not be confirmed for the DSS where no difference was found (median 16 mo for both groups).

Concerning the development of distant metastases we could not obtain the same results as stated in several studies. ${ }^{6,20,23}$ In our analysis the rate of distant failure was not lower in patients with radio-/chemotherapy as in patients receiving RT alone. The reason might be that especially in the higher dose groups, the majority of patients receiving chemotherapy had stage IIIB whereas in the exclusive radiotherapy groups, also stages I and II were included. The LCR at $1 y$ was in all 4 treatment groups nearly the same with mean $45 \%$ and no advantage for higher radiation doses could be observed. Probably the number of patients was too small. The LCR at $2 \mathrm{y}$ was low in all four groups with the poorest result (15\%) for the group receiving 50-60 Gy without chemotherapy.

To improve the local control, different efforts have been made in the last years. Developments in 3D-planning and new techniques like self-gated radiotherapy at deep-inspiration breath hold (DIBH) enable a better tumour targeting and sparing of normal tissue which allows an escalation of the radiation dose up to $100 \mathrm{~Gy}$. Local control rates of $50-80 \%$ have been reported with radiation doses varying from 70.2 to $90 \mathrm{~Gy} .3,8,13,17,24,25$ The limiting factor for dose escalation is the surrounding normal lung tissue. Radiation doses of $\geq 90$ Gy lead to severe pulmonary toxicity. ${ }^{3,13}$ Several studies showed that intensity modulated radiotherapy (IMRT), especially if guided by PET/CT imaging, has the ability to spare more normal lung tissue and allows $25-30 \%$ higher doses than with 3D-conformal radio- therapy. ${ }^{26,27}$ Advances in tumour staging could result in smaller treatment volumes, which would enable the application of higher radiation doses. Especially PET/CT improves the detection of lymphnode metastases and the differentiation between tumour tissue and atelectasis. De Ruysscher et al. showed in a planning study that the use of a combined PET/CT simulator reduced the radiation dose to normal lung tissue and oesophagus and thus allowed a significant radiation dose escalation. ${ }^{28}$

Still, the risk of normal tissue toxicity should not be underestimated ${ }^{3,27}$ and new trials to evaluate a safe dose-escalation technique are obligatory.

\section{Conclusions}

The low incidence of severe side effects confirms that the application radiation doses up to $70 \mathrm{~Gy}$ with 3D-conformal radiotherapy is feasible and safe, taking into account 3D dose volume constraints. Our work suggests that the combination of chemotherapy and 3D-conformal radiotherapy of 66-70 Gy prolongs moderately the overall survival for patients with inoperable non-small-cell lung cancer with a low risk of severe pneumonitis.

Nevertheless, local control rates remain low, especially after $2 \mathrm{y}$. Thus the possibility of further dose escalation also in more advanced tumour stages with a special regard to the long-term pulmonary and oesophageal toxicity should be investigated.

\section{Acknowledgements}

The authors would like to thank Martha Beranek for her technical support in calculating and retrieving dose-volume histograms and Brigitte Keringer and Waltraud Stepan for their secretarial assistance. 


\section{References}

1. Ferlay J, Bray F, Sankila R, Parkin DM. EUCAN: Cancer incidence, Mmortality and prevalence in the European Union 1997. Version 4.0. IARC Cancer Base No. 4. Lyon: IARC; 1999.

2. Bray F, Tycynski JE, Parkin DM. Going up or coming down? The changing phases of lung cancer epidemic from 1967 to 1999 in the 15 European Union countries. Eur J Cancer 2004; 40: 96-125.

3. Bradley J, Graham MV, Winter K, Purdy JA, Komaki R, Roa WH, et al. Toxicity and outcome results of RTOG 9311: a phase I-II dose-escalation study using three-dimensional conformal radiotherapy in patients with inoperable non-small cell lung carcinoma. Int J Radiat Oncol Biol Phys 2005; 61: 318-28.

4. Semrau S, Bier A, Thierbach U, Virchow C, Ketterer P, Klautke G, et al. R. 6-Year Experience of concurrent radiochemotherapy with vinorelbine plus a platinum compound in multimorbid or aged patients with inoperable Non-Small Cell Lung Cancer. Strahlenther Onkol 2007; 183: 30-5.

5. Sim S, Rosenzweig KE, Schindelheim R, Ng KK, Leibel SA. Induction chemotherapy plus three-dimensional conformal radiation therapy in the definitive treatment of locally advanced non-smallcell lung cancer. Int J Radiat Oncol Biol Phys 2001; 51: $660-5$.

6. Novello S, Le Chevalier T. Use of chemo-radiotherapy in locally advanced non-small cell lung cancer. Eur J Cancer 2002; 38: 292-9.

7. Ball D, Matthews J, Worotniuk V, Crennan E. Longer survival with higher doses of thoracic radiotherapy in patients with limited non-small cell lung cancer. Int J Radiat Oncol Phys 1993; 25: 599-604.

8. Thirion P, Holmberg O, Collins C, O'Shea C, Moriarty M, Pomeroy M, et al. Escalated dose for non-small lung cancer with accelerated hypofractionated three-dimensional conformal radiation therapy. Radiother Oncol 2004; 71: 163-6.

9. Willner J, Baier K, Caragiani E, Tschammler A, Flentje M. Dose, volume and tumor control predictions in primary radiotherapy of non-small-cell lung cancer. Int J Radiat Oncol Biol Phys 2002; 52: 382-9.
10. Baumann M, Herrmann T, Matthiessen, Koch R, Strelocke K, Paul U. CHARTWEL-Bronchus (ARO 97-1): a randomized multicenter trial to compare conventional fractionated radiotherapy with CHARTWEL radiotherapy in inoperable nonsmall-call bronchial carcinoma. Strahlenther Onkol 1997; 173: 663-7.

11. Catalano G, Jereczek-Fossa B, De Pas T, Leon ME, Cattani F, Spaggiari L, et al. Three-times daily radiotherapy with induction chemotherapy in locally advanced non-small cell lung cancer. Strahlenther Onkol 2005; 181: 363-71.

12. Rengan R, Rosenzweig KE, Venkatraman E et al. Improved local control with higher doses of radiation in large-volume stage III non-small-cell lung cancer. Int J Radiat Oncol Biol Phys 2004; 60(3): 741-747

13. Rosenzweig KE, Fox JL, Yorke E, Amols H, Jackson A, Rusch V, et al. Results of a phase I dose-escalation study using three-dimensional conformal radiotherapy in the treatment of inoperable nonsmall lung carcinoma. Cancer 2005; 103: 2118-27.

14. International Commission on Radiation Units and Measurements. Prescribing, recording and reporting photon beam therapy. ICRU Report 50 . Bethesda: International Commission on Radiation Units and Measurements; 1993.

15. Graham MV, Purdy JA, Emami B, Harms W, Bosch W, Lockett MA, et al. Clinical dose-volume histogram analysis of pneumonitis after 3D treatment for non small cell lung cancer (NSCLC). Int J Radiat Oncol Biol Phys 1999; 45: 323-9.

16. Cox JD, Stretz J, Pajak TF. Toxicity criteria of the Radiation Therapy Oncology Group (RTOG) and the European Organisation for Research and Treatment of cancer (EORTC). Int J Radiat Oncol Biol Phys 1995; 31: 1341-6.

17. Kaplan EL, Meier P. Non parametric estimation for incomplete observations. J Am Stat Ass 1958; 53: 457-81.

18. Belderbos J, Heemsbergen W, De Jaeger K, Baas $\mathrm{P}$, Lebesque JV. Final results of a phase I/II dose escalation trial in non-small cell lung cancer using three-dimensional conformal radiotherapy. Int $J$ Radiat Oncol Biol Phys 2006; 66: 126-34.

19. Kong FM, Hayman JA, Griffith KA, Kalemkerian GP, Arenberg D, Lyons S, et al. Final toxicity results of a radiation-dose escalation study on patients with non-small-cell lung cancer (NSCLC): Predictors for radiation pneumonitis and fibrosis. Int J Radiat Oncol Biol Phys 2006; 65: 1075-86 
20. Dillman RO, Seagren SL, Propert KJ, Guerra J, Eaton WL, Perry MC, et al. A randomized trial of induction chemotherapy plus high-dose radiation versus radiation alone in stage III non-small-cell lung cancer. N Engl J Med 1990; 323: 940-5.

21. Furuse K, Kubota K, Kawahara M, Kodama N, Ogawara M, Akira M, et al. Phase II study of concurrent radiotherapy and chemotherapy for unresectable stage III non small cell lung cancer. Southern Osaka Lung Cancer Study Group. J Clin Oncol 1995; 13: 869-75.

22. Kubota K, Furuse K, Kawahara M, Kodama N, Yamamoto M, Ogawara M, et al. Role of radiotherapy in combined modality treatment of locally advanced non-small-cell lung cancer. J Clin Oncol 1994; 12: 1547-52.

23. Le Chevalier T, Arriagada R, Quoix E, Ruffie P, Martin M, Tarayre M, et al. Radiotherapy alone versus combined chemotherapy and radiotherapy in nonresectable non-small-cell lung cancer: first analysis of a randomized trial in 353 patients. $J$ Natl Cancer Inst 1991; 83: 417-23.

24. Barnes E, Murray B, Robinson D, Underwood LJ, Hanson J, Roa WH. Dosimetric evaluation of lung tumour immobilization using breath hold at deep inspiration. Int J Radiat Oncol Biol Phys 2001; 50: 1091-8.

25. Hof H, Herfarth KK, Munter M, Essig M, Wannenmacher M, Debus J. The use of the multislice CT for the determination of respiratory lung tumor movement in stereotactic single-dose irradiation. Strahlenther Onkol 2003; 179: 542-7.

26. Grills IS, Yan D, Martinez AA, Vicini FA, Wong JW, Kestin LL. Potential for reduced toxicity and does escalation in the treatment of inoperable non-small-cell lung cancer: a comparison of intensity-modulated radiation therapy (IMRT), 3D conformal radiation, and elective nodal irradiation. Int J Oncol Biol Phys 2003; 57: 875-90.

27. Holloway CL, Robinson D, Murray B, Amanie J, Butts C, Smylie M, et al. Results of a phase I study to dose escalate using intensity modulated radiotherapy guided by combined PET/CT imaging with induction chemotherapy for patients with non-small cell lung cancer. Radiother Oncol 2004; 73: 285-97.

28. De Ruysscher D, Wanders S, Minken A, Lumens A, Schiffelers J, Stultiens C, et al. Effects of radiotherapy planning with dedicated combined PET$\mathrm{CT}$ simulator of patients with non-small cell lung cancer on dose limiting normal tissues and radiation dose-escalation: a planning study. Radiother Oncol 2005; 77: 5-10. 